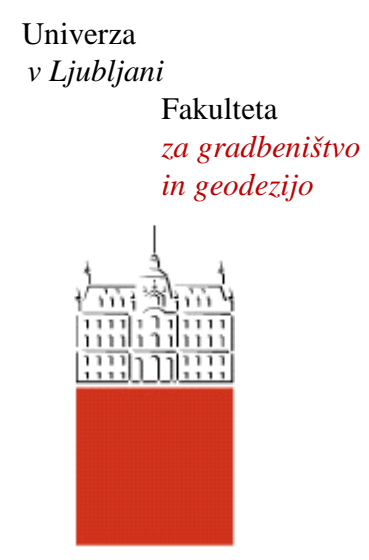

Jamova 2

1000 Ljubljana, Slovenija http://www3.fgg.uni-lj.si//

DRUGG - Digitalni repozitorij UL FGG http://drugg.fgg.uni-lj.si/

Ta članek je avtorjeva zadnja recenzirana različica, kot je bila sprejeta po opravljeni recenziji.

Prosimo, da se pri navajanju sklicujte na bibliografske podatke, kot je navedeno:
University
of Ljubljana

Faculty of

Civil and Geodetic

Engineering

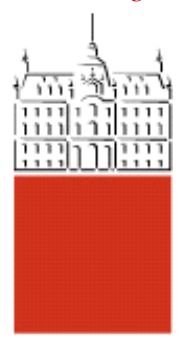

Jamova 2

SI - 1000 Ljubljana, Slovenia

http://www3.fgg.uni-lj.si/

DRUGG - The Digital Repository http://drugg.fgg.uni-lj.si/

This version of the article is author's manuscript as accepted for publishing after the review process.

When citing, please refer to the publisher's bibliographic information as follows:

Brank, B. 2003. On composite shell models with a piecewise linear warping function. Compos. struct. 59, 2: 163-171.

http://www.journals.elsevier.com/composite-structures/ 


\title{
On composite shell models with a piecewise linear warping function
}

\author{
Boštjan Brank
}

Faculty of Civil and Geodetic Engineering, University of Ljubljana, Jamova 2, 1000 Ljubljana, Slovenia

\begin{abstract}
A multilayered shell model accounting for a piecewise linear (i.e. zig-zag) distribution of displacements through the laminate thickness is discussed. The model has seven unknown kinematic variables: three displacements of the middle surface, two rotations of the shell director and two displacements associated with the wrinkling of the laminate crosssections. The initial transverse shear stress field is introduced, and the constitutive relations are then relaxed in the framework of the variational principle. Finite element solutions obtained with this kind of model are compared with the analytical solutions for the case of cylindrical shell bending.
\end{abstract}

Keywords: Composite shell; Finite element; Zig-zag function; Interlaminar continuity

\section{Introduction}

Many theories for laminated composite plates and shells can be found in the composite mechanics literature. They are usually classified into three groups: (i) classical theories based on the Kirchhoff kinematic hypothesis (e.g. [17]), (ii) first-order shear deformation theories based on the Mindlin kinematic hypothesis (e.g. [17]) and (iii) higher-order theories with refined throughthe-thickness variation of displacements (see e.g. review of Noor et al. [16]). Two different approaches have been basically explored for the higher-order theories: one based on an equivalent single-layer model and the other one on a layerwise model.

This work is restricted to a single-layer shell model, which basic characteristic is a piecewise linear warping function. The plate model with such zig-zag displacement function was introduced by Di Scuva (e.g. [19]) and was later examined and modified by many others, e.g. Murakami [15], Toledano and Murakami [20], Carrera [9], among others. Some researchers exploited the idea also to cylindrical shell problems: e.g. $[2,11,14,18]$. Both the plate and the cylindrical shell solutions were typically obtained in an analytic manner by using Fourier series. In contrast to the analytical ap- proach the finite element implementation has been mainly restricted to the plate formulations (e.g. $[8,10,13])$ and only very few examples of general shell elements can be found in the literature $([5,12])$. This can be due to a relatively complicated finite element implementation procedure.

It is an aim of this paper to revisit the theoretical formulation for multilayered composite shells and present it in a very clear form in order to provide a basis for different possible finite element implementations. We focus on a formulation which assumes: (i) a zig-zag distribution of displacements through the laminate thickness and (ii) a layerwise independent quadratic distribution of transverse shear stresses in the framework of Reissner's functional. The pertinent features of this kind of shell model are:

- the number of unknown kinematic variables is independent on a number of layers,

- the transverse shear stresses and stress resultants are obtained from the constitutive equations and not from a post-processing,

- a sharp change of displacement slope at layer interfaces (the fact known from the 3-d analytical solutions of composite plates and shells) is taken into account,

- equilibrium conditions are satisfied at layer interfaces,

- there is no need to use shear correction factors. 


\section{Higher-order theory for multilayered shells with seven parameters}

This section firstly elaborates upon a hypothesis which refines through-the-thickness variation of displacements by using a piecewise linear warping function, the so-called zig-zag function. We then develop the corresponding Green-Lagrange strain measures and the stress resultants. Finally, a trial distribution of the transverse shear stresses through a laminate thickness is introduced, and the variational principle is used to relax the constitutive equations and satisfy the interlaminar equilibrium constraints.

\subsection{Assumed displacements}

An enriched kinematic field is developed with respect to the Reissner-Mindlin kinematics in order to better predict through-the-thickness variation of displacements in laminated shells. To that end, the position vector from the initial configuration

$\mathbf{X}\left(\xi^{1}, \xi^{2}, \xi\right)=\mathbf{X}^{0}\left(\xi^{1}, \xi^{2}\right)+\xi \mathbf{T}\left(\xi^{1}, \xi^{2}\right)$

is transformed into its counterpart at the deformed configuration as

$\mathbf{x}\left(\xi^{1}, \xi^{2}, \xi\right)=\mathbf{x}^{0}\left(\xi^{1}, \xi^{2}\right)+\xi \mathbf{t}\left(\xi^{1}, \xi^{2}\right)+f(\xi) \mathbf{w}\left(\xi^{1}, \xi^{2}\right)$

where the last term introduces a zig-zag variation of the displacements through-the-laminate thickness. In (1) and (2) $\left(\xi^{1}, \xi^{2}\right) \in \mathscr{A}$ are the curvilinear coordinates, $\mathscr{A}$ is the domain of the shell middle surface parametrization, $\xi \in[-h / 2, h / 2]$ is the through-the-thickness coordinate, $h$ is the shell thickness, $\mathbf{X}^{0}$ and $\mathbf{x}^{0}$ are the middle surface position vectors, $\mathbf{T}$ and $\mathbf{t}$ are the shell directors, and $\mathbf{w}$ is the displacement vector related to the wrinkling of the laminate cross-sections. We assume the shell director vector to be of unit length

$\|\mathbf{T}\|=\|\mathbf{t}\|=1$

and perpendicular to the middle surface at the initial configuration. Its motion can be then completely defined by two rotations $\alpha^{1}$ and $\alpha^{2}$ (see e.g. [4] for different possible choices) so that we can write

$\mathbf{t}=\mathbf{t}\left(\alpha^{1}, \alpha^{2}\right)$

Function $f(\xi)$ in (2) is a zig-zag (piecewise linear) function which is at layer $K \in[1, N]$ ( $N$ is the total number of layers) given as ${ }^{1}$

\footnotetext{
${ }^{1}$ The zig-zag function defined in (5) is in general having different slope at different layers depending on the layer's height. It is also possible to define zig-zag function with slopes remaining the same in all layers (see e.g. [11])
}

$f\left(\xi_{K}\right)=(-1)^{K} \frac{\xi_{K}}{h_{K} / 2}$

where $\xi_{K} \in\left[-h_{K} / 2, h_{K} / 2\right]$ is a local through-the-thickness coordinate and $h_{K}$ is the layer's thickness. Relation between $\xi_{K}$ and $\xi$ coordinates is

$\xi=\xi_{K}+\xi^{K 0}$

where $\xi^{K 0}$ is the value of $\xi$ in the middle of the layer $K$ (i.e. at $\xi_{K}=0$ ). We assume that layer 1 begins at $\xi=$ $-h / 2$.

The above concepts, which are illustrated in Fig. 1 for a four-layered laminate, can be viewed as a direct refinement of the first-order shear-deformation theory as shown by Brank and Carrera [6].

\subsection{Strains}

When defining strains it is suitable to locally replace curvilinear coordinates $\xi^{1}$ and $\xi^{2}$, introduced in (1) and (2), with Cartesian coordinates $\hat{x}_{1}$ and $\hat{x}_{2}$. To that end we
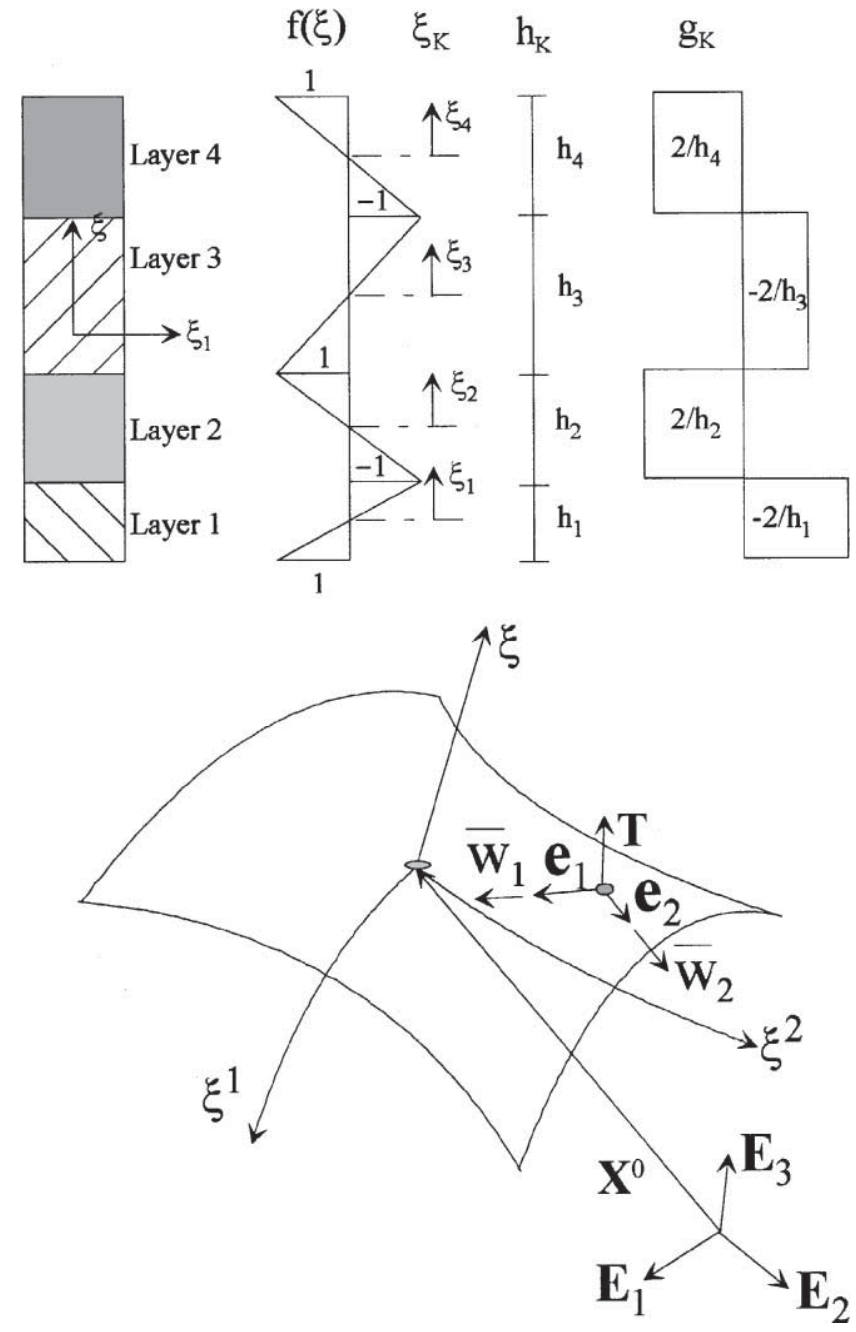

Fig. 1. Warping function and displacements $\tilde{w}_{1}$ and $\tilde{w}_{2}$. 
define on the middle surface point ${ }^{2}$ at the initial configuration a local Cartesian basis $\mathbf{e}_{i}$ such that

$\mathbf{e}_{3}=\mathbf{T}, \quad \mathbf{e}_{1} \perp \mathbf{e}_{3}, \quad \mathbf{e}_{2} \perp \mathbf{e}_{1}$

Transformation from the curvilinear coordinates $\xi^{1}$ and $\xi^{2}$ to the local Cartesian ones has the following form (see e.g. [3])

$\left\{\begin{array}{l}\frac{\partial}{\partial \hat{x}_{1}} \\ \frac{\partial}{\partial \hat{x}_{2}}\end{array}\right\}=\left[\begin{array}{ll}\mathbf{X}_{, \xi^{1}}^{0} \cdot \mathbf{e}_{1} & \mathbf{X}_{, \xi^{1}}^{0} \cdot \mathbf{e}_{2} \\ \mathbf{X}_{, \xi^{2}}^{0} \cdot \mathbf{e}_{1} & \mathbf{X}_{, \xi^{2}}^{0} \cdot \mathbf{e}_{2}\end{array}\right]^{-1}\left\{\begin{array}{l}\frac{\partial}{\partial \xi^{1}} \\ \frac{\partial}{\partial \xi^{2}}\end{array}\right\}$

where $(\circ)_{\xi^{\alpha}}$ are partial derivatives with respect to the coordinate $\xi^{\alpha}$. Note that $\hat{x}_{3}=\xi$.

The choice of displacements (2) leads, with transformation (8), to the following vector basis in the shell deformed configuration

$\mathbf{a}_{\alpha}=\mathbf{x}_{, \alpha}=\mathbf{x}_{, \alpha}^{0}+\xi \mathbf{t}_{, \alpha}+f \mathbf{w}_{, \alpha}$

$\mathbf{a}_{3}=\mathbf{t}+g \mathbf{w}$

where $(0)_{\alpha}=\partial(0) / \partial \hat{x}_{\alpha}$ and $g$ is derivative of $f$, see (5), with respect to the $\xi$ coordinate

$g=\frac{\mathrm{d} f}{\mathrm{~d} \xi} \Rightarrow$ for layer $K$ we have $g_{K}=(-1)^{K} 2 / h_{K}$

The base vectors at the initial configuration follow from (1) as

$$
\begin{aligned}
& \mathbf{g}_{\alpha}=\mathbf{X}_{, \alpha}=\mathbf{X}_{, \alpha}^{0}+\xi \mathbf{T}_{, \alpha} \\
& \mathbf{g}_{3}=\mathbf{T}
\end{aligned}
$$

The Green-Lagrange strains with respect to the local Cartesian frame are then defined as

$E_{i j}=\frac{1}{2}\left(\mathbf{a}_{i} \cdot \mathbf{a}_{j}-\mathbf{g}_{i} \cdot \mathbf{g}_{j}\right)$

with their explicit forms given as

$$
\begin{aligned}
& E_{\alpha \beta}=\frac{1}{2}\left(\mathbf{x}_{, \alpha}^{0} \cdot \mathbf{x}_{, \beta}^{0}-\mathbf{X}_{, \alpha}^{0} \cdot \mathbf{X}_{, \beta}^{0}\right)+\frac{1}{2} \xi\left(\mathbf{x}_{, \alpha}^{0} \cdot \mathbf{t}_{, \beta}+\mathbf{x}_{, \beta}^{0} \cdot \mathbf{t}_{, \alpha}\right. \\
& \left.-\mathbf{X}_{, \alpha}^{0} \cdot \mathbf{T}_{, \beta}-\mathbf{X}_{, \beta}^{0} \cdot \mathbf{T}_{, \alpha}\right)+\frac{1}{2} f\left(\mathbf{x}_{, \alpha}^{0} \cdot \mathbf{w}_{, \beta}+\mathbf{x}_{, \beta}^{0} \cdot \mathbf{w}_{, \alpha}\right) \\
& +\underline{\frac{1}{2} \xi^{2}\left(\mathbf{t}_{, \alpha} \cdot \mathbf{t}_{, \beta}-\mathbf{T}_{, \alpha} \cdot \mathbf{T}_{, \beta}\right)}+\underline{\underline{1}} \xi f\left(\mathbf{t}_{, \alpha} \cdot \mathbf{w}_{, \beta}-\mathbf{t}_{, \beta} \cdot \mathbf{w}_{, \alpha}\right) \\
& +\frac{1}{2} f^{2} \mathbf{w}_{, \alpha} \cdot \mathbf{w}_{, \beta} \\
& E_{\alpha 3}=\frac{1}{2} \mathbf{x}_{, \alpha}^{0} \cdot \mathbf{t}+\frac{1}{2} g \mathbf{x}_{, \alpha}^{0} \cdot \mathbf{w}+\underline{\frac{1}{2} f \mathbf{w}_{, \alpha} \cdot \mathbf{t}}+\underline{\frac{1}{2} f g \mathbf{w}_{, \alpha} \cdot \mathbf{w}} \\
& +\underline{\frac{1}{2} \xi g \mathbf{t}_{, \alpha} \cdot \mathbf{w}} \\
& E_{33}=\underline{g \mathbf{t} \cdot \mathbf{w}}+\underline{\frac{1}{2} g^{2} \mathbf{w} \cdot \mathbf{w}}
\end{aligned}
$$

The underlined terms in the above expressions are nonlinear. In the following we will concentrate only on a linear model, which is naturally based on linear strains. It is therefore necessary to exclude all nonlinear strains from (13)-(15).

To simplify the notation we define the following two vectors

\footnotetext{
${ }^{2}$ In the finite element implementation the local Cartesian frames need only be constructed at the element nodes and at the numerical integration points.
}

$$
\begin{aligned}
& \mathbf{E}_{\mathrm{n}}=\left[E_{11}, E_{22}, 2 E_{12}\right]^{\mathrm{T}} \\
& \mathbf{E}_{\mathrm{s}}=\left[2 E_{13}, 2 E_{23}\right]^{\mathrm{T}}
\end{aligned}
$$

where each of them is composed as

$$
\begin{aligned}
& \mathbf{E}_{\mathrm{n}}=\boldsymbol{\varepsilon}+\xi \boldsymbol{\kappa}+f \tilde{\boldsymbol{\kappa}} \\
& \mathbf{E}_{\mathrm{s}}=\boldsymbol{\gamma}+g \tilde{\boldsymbol{\gamma}}
\end{aligned}
$$

The vectors $\boldsymbol{\varepsilon}=\left[\varepsilon_{11}, \varepsilon_{22}, 2 \varepsilon_{12}\right]^{\mathrm{T}}, \boldsymbol{\kappa}=\left[\kappa_{11}, \kappa_{22}, 2 \kappa_{12}\right]^{\mathrm{T}}$ and $\gamma=\left[\gamma_{13}, \gamma_{23}\right]^{\mathrm{T}}$ collect the linear membrane, the linear bending and the linear transverse shear strains of the classical Reissner-Mindlin shell model, while the vectors $\tilde{\boldsymbol{\kappa}}=\left[\tilde{\kappa}_{11}, \tilde{\kappa}_{22}, 2 \tilde{\kappa}_{12}\right]^{\mathrm{T}}$ and $\tilde{\boldsymbol{\gamma}}=\left[\tilde{\gamma}_{13}, \tilde{\gamma}_{23}\right]^{\mathrm{T}}$ collect additional linear strains due to enriched kinematics. Explicit strain expressions follow directly from (13)-(15)

$$
\begin{aligned}
& \varepsilon_{\alpha \beta}=\frac{1}{2}\left(\mathbf{x}_{, \alpha}^{0} \cdot \mathbf{x}_{, \beta}^{0}-\mathbf{X}_{, \alpha}^{0} \cdot \mathbf{X}_{, \beta}^{0}\right) \\
& \kappa_{\alpha \beta}=\frac{1}{2}\left(\mathbf{x}_{, \alpha}^{0} \cdot \mathbf{t}_{, \beta}+\mathbf{x}_{, \beta}^{0} \cdot \mathbf{t}_{, \alpha}-\mathbf{X}_{, \alpha}^{0} \cdot \mathbf{T}_{, \beta}-\mathbf{X}_{, \beta}^{0} \cdot \mathbf{T}_{, \alpha}\right) \\
& \gamma_{\alpha 3}=\mathbf{x}_{, \alpha}^{0} \cdot \mathbf{t} \\
& \tilde{\kappa}_{\alpha \beta}=\frac{1}{2}\left(\mathbf{x}_{, \alpha}^{0} \cdot \mathbf{w}_{, \beta}+\mathbf{x}_{, \beta}^{0} \cdot \mathbf{w}_{, \alpha}\right) \\
& \tilde{\gamma}_{\alpha 3}=\mathbf{x}_{, \alpha}^{0} \cdot \mathbf{w}
\end{aligned}
$$

It is further assumed that the refined displacement vector $\mathbf{w}$ lies in a plane tangent to the middle surface, which leads to

$\mathbf{T} \cdot \mathbf{w}=0$

Introducing $\tilde{w}_{i}$ (components of $\mathbf{w}$ with respect to the local Cartesian basis $\mathbf{e}_{i}$ ) and $w_{i}$ (their counterparts in the global coordinate system), and noting from (20) and (7) that $\tilde{w}_{3}=0$, it can be concluded that the transformation rule has a form

$w_{i}=Q_{i \alpha} \tilde{w}_{\alpha}, \quad\left[Q_{i \alpha}\right]=\left[\mathbf{e}_{1}, \mathbf{e}_{2}\right]$

Eqs. (2), (4) and (21) indicate that the present model has seven kinematic parameters: three displacements of the middle surface $\left(\mathbf{u}^{0}=\mathbf{x}^{0}-\mathbf{X}^{0}\right)$ defined in the global coordinate system, two rotations of the shell director ${ }^{3}$ $\left(\alpha^{1}, \alpha^{2}\right)$, and two displacements related to the warping of the laminate cross-sections $\left(\tilde{w}_{1}, \tilde{w}_{2}\right)$ defined in a local coordinate system

$\mathbf{u}=\left[\mathbf{u}^{0}, \alpha^{1}, \alpha^{2}, \tilde{w}_{1}, \tilde{w}_{2}\right]^{\mathrm{T}}$

We note again that this number is independent on the total number of layers.

\subsection{Stress resultants and constitutive relations}

Having defined strains of the chosen model we can proceed with the constitutive relations. For that purpose let us first collect stresses (defined with respect to the local Cartesian basis) in two vectors as

\footnotetext{
${ }^{3}$ Rotations are usually chosen such that they rotate around the local $\mathbf{e}_{1}$ and $\mathbf{e}_{2}$ axes.
} 


$$
\begin{aligned}
& \boldsymbol{\sigma}_{\mathrm{n}}=\left[\boldsymbol{\sigma}^{11}, \boldsymbol{\sigma}^{22}, \boldsymbol{\sigma}^{12}\right]^{\mathrm{T}} \\
& \boldsymbol{\sigma}_{\mathrm{s}}=\left[\boldsymbol{\sigma}^{13}, \boldsymbol{\sigma}^{23}\right]^{\mathrm{T}}
\end{aligned}
$$

where the in-plane stresses are collected in $\sigma_{\mathrm{n}}$, and $\boldsymbol{\sigma}_{\mathrm{s}}$ contains the transverse shear stresses. In a layer $K \in$ $[1, N]$, which is made of an orthotropic material, we have the following constitutive relations

$$
\begin{aligned}
& \boldsymbol{\sigma}_{\mathrm{n}}=\mathbf{C}_{K} \mathbf{E}_{\mathrm{n}} \\
& \boldsymbol{\sigma}_{\mathrm{s}}=\mathbf{C}_{K}^{\mathrm{s}} \mathbf{E}_{\mathrm{s}}
\end{aligned}
$$

where explicit expressions for matrices $\mathbf{C}_{K}$ and $\mathbf{C}_{K}^{\mathrm{s}}$ can be found in the textbooks or e.g. in Brank, Perić and Damjanić [3]. They depend on six independent material constants and the transformation from the material to the local Cartesian coordinates.

The internal virtual work

$$
\begin{aligned}
\delta \Pi= & \int_{A} \int_{-h / 2}^{h / 2}\left(\boldsymbol{\sigma}_{\mathrm{n}} \cdot \delta \mathbf{E}_{\mathrm{n}}+\boldsymbol{\sigma}_{\mathrm{s}} \cdot \delta \mathbf{E}_{\mathrm{s}}\right) \mu \mathrm{d} \xi \mathrm{d} A \\
= & \int_{A} \int_{-h / 2}^{h / 2}\left[\boldsymbol{\sigma}_{\mathrm{n}} \cdot(\delta \boldsymbol{\varepsilon}+\xi \delta \boldsymbol{\kappa}+f \delta \tilde{\boldsymbol{\kappa}})\right. \\
& \left.+\boldsymbol{\sigma}_{\mathrm{s}} \cdot(\delta \boldsymbol{\gamma}+g \delta \tilde{\boldsymbol{\gamma}})\right] \mu \mathrm{d} \xi \mathrm{d} A \\
= & \int_{A}[(\mathbf{n} \cdot \delta \boldsymbol{\varepsilon}+\mathbf{m} \cdot \delta \boldsymbol{\kappa}+\mathbf{q} \cdot \delta \boldsymbol{\gamma})+(\tilde{\mathbf{m}} \cdot \delta \tilde{\boldsymbol{\kappa}}+\tilde{\mathbf{q}} \cdot \delta \tilde{\boldsymbol{\gamma}})] \mathrm{d} A
\end{aligned}
$$

where $\delta(\circ)=D(\circ) \cdot \delta \mathbf{u}$ are variations of strains, provides definition for the membrane, the bending and the transverse shear forces. The differential element of the shell volume, $\mathrm{d} V$, is related to the differential element of the shell middle surface, $\mathrm{d} A$, as

$\mathrm{d} V=\mu \mathrm{d} \xi \mathrm{d} A$

where $\mu=\sqrt{g} / \sqrt{g^{0}}$, with $g=\operatorname{det}\left[\mathbf{X}_{, \alpha} \cdot \mathbf{X}_{, \beta}\right] \quad$ and $g^{0}=\operatorname{det}\left[\mathbf{X}_{, \alpha}^{0} \cdot \mathbf{X}_{, \beta}^{0}\right]$. The following stress resultants are identified as the membrane, the bending and the transverse shear forces of the classical Reissner-Mindlin model

$$
\begin{aligned}
& \mathbf{n}=\sum_{K=1}^{N} \int_{-h_{K} / 2}^{h_{K} / 2} \boldsymbol{\sigma}_{\mathrm{n}} \mu \mathrm{d} \xi_{K}, \quad \mathbf{m}=\sum_{K=1}^{N} \int_{-h_{K} / 2}^{h_{K} / 2} \boldsymbol{\sigma}_{\mathrm{n}} \xi \mu \mathrm{d} \xi_{K} \\
& \mathbf{q}=\sum_{K=1}^{N} \int_{-h_{K} / 2}^{h_{K} / 2} \boldsymbol{\sigma}_{\mathrm{s}} \mu \mathrm{d} \xi_{K}
\end{aligned}
$$

while the enrichment of the kinematic field produces additional stress resultants

$$
\tilde{\mathbf{m}}=\sum_{K=1}^{N} \int_{-h_{K} / 2}^{h_{K} / 2} \boldsymbol{\sigma}_{\mathrm{n}} f_{K} \mu \mathrm{d} \xi_{K}, \quad \tilde{\mathbf{q}}=\sum_{K=1}^{N} \int_{-h_{K} / 2}^{h_{K} / 2} \boldsymbol{\sigma}_{\mathrm{s}} g_{K} \mu \mathrm{d} \xi_{K}
$$

Having defined stress resultants in (27) and (28), and stress-strain relations in (24), we can proceed with de- fining the laminate stiffness. It can be shown that the following matrix relation can be obtained from (27), (28), (24) and (17)

$$
\left\{\begin{array}{c}
\mathbf{n} \\
\mathbf{m} \\
\mathbf{q} \\
\tilde{\mathbf{m}} \\
\tilde{\mathbf{q}}
\end{array}\right\}=\left[\begin{array}{ccccc}
\mathbf{C}_{0} & \mathbf{C}_{1} & \mathbf{0} & \mathbf{F}_{10} & \mathbf{0} \\
\mathbf{C}_{1} & \mathbf{C}_{2} & \mathbf{0} & \mathbf{F}_{11} & \mathbf{0} \\
\mathbf{0} & \mathbf{0} & \mathbf{Z}_{0} & \mathbf{0} & \mathbf{Z}_{1} \\
\mathbf{F}_{10} & \mathbf{F}_{11} & \mathbf{0} & \mathbf{F}_{02} & \mathbf{0} \\
\mathbf{0} & \mathbf{0} & \mathbf{Z}_{1} & \mathbf{0} & \mathbf{Z}_{2}
\end{array}\right]\left\{\begin{array}{c}
\boldsymbol{\varepsilon} \\
\boldsymbol{\kappa} \\
\boldsymbol{\gamma} \\
\tilde{\boldsymbol{\kappa}} \\
\tilde{\gamma}
\end{array}\right\}
$$

The submatrices in (29) above are given as

$$
\begin{aligned}
\mathbf{C}_{J} & =\sum_{K=1}^{N_{\text {lay }}} \int_{-h_{K} / 2}^{h_{K} / 2} \mathbf{C}_{K} \xi^{J} \mu \mathrm{d} \xi_{K} \\
\mathbf{Z}_{J} & =\sum_{K=1}^{N_{\text {lay }}} \int_{-h_{K} / 2}^{h_{K} / 2} \mathbf{C}_{K}^{\mathrm{s}}\left(g_{K}\right)^{J} \mu \mathrm{d} \xi_{K} \\
\mathbf{F}_{I J} & =\sum_{K=1}^{N_{\text {lay }}} \int_{-h_{K} / 2}^{h_{K} / 2} \mathbf{C}_{K} \xi^{I}\left(f_{K}\right)^{J} \mu \mathrm{d} \xi_{K}, \quad J=0,1,2 ; I=0,1
\end{aligned}
$$

where $\mathbf{F}_{I J}, \mathbf{Z}_{1}$ and $\mathbf{Z}_{2}$ appear due to enriched kinematics.

\subsection{Reissner's functional}

In order to improve an effect of the transverse shear deformation, to satisfy the continuity of the transverse shear stresses across the layer interfaces and to avoid usage of the transverse shear correction factors, we use the Reissner's functional in the following form

$$
\begin{aligned}
\delta & \Pi_{\mathrm{R}}\left(\mathbf{u}, \boldsymbol{\sigma}_{\mathrm{s}} ; \delta \mathbf{u}, \delta \boldsymbol{\sigma}_{\mathrm{s}}\right) \\
= & \int_{A}[\delta \boldsymbol{\varepsilon} \cdot \mathbf{n}+\delta \boldsymbol{\kappa} \cdot \mathbf{m}+\delta \boldsymbol{\gamma} \cdot \mathbf{q}+\delta \tilde{\boldsymbol{\kappa}} \cdot \tilde{\mathbf{m}}+\delta \tilde{\boldsymbol{\gamma}} \cdot \tilde{\mathbf{q}}] \mathrm{d} A \\
& +\int_{A}\left\{\sum_{K=1}^{N} \int_{h_{K} / 2}^{h_{K} / 2} \delta \boldsymbol{\sigma}_{\mathrm{s}} \cdot\left[-\left[\mathbf{C}_{K}^{\mathrm{s}}\right]^{-1} \boldsymbol{\sigma}_{\mathrm{s}}+\left(\boldsymbol{\gamma}+g_{K} \tilde{\boldsymbol{\gamma}}\right)\right] \mu \mathrm{d} \xi_{K}\right\} \mathrm{d} A \\
& -\left.\int_{A^{-}} \mathbf{p}^{-} \cdot \delta \mathbf{u}\right|_{-h / 2} \mathrm{~d} A^{-}-\left.\int_{A^{+}} \mathbf{p}^{+} \cdot \delta \mathbf{u}\right|_{h / 2} \mathrm{~d} A^{+}=0
\end{aligned}
$$

where we assume that the composite shell is pressured by $\mathbf{p}^{-}$on its inner surface $A^{-}$and by $\mathbf{p}^{+}$on its outer surface $A^{+}$. Matrix $\left[\mathbf{C}_{K}^{\mathrm{s}}\right]^{-1}$ is part of the layer $K$ compliance matrix associated with the transverse shear deformations.

\subsection{Transverse shear stresses}

Functional (31) allows for an assumed distribution of the transverse shear stresses through the laminate layers. In this work we examine quadratic variation through each layer, although other assumptions are also possible (e.g. linear, qubic, etc.). The stress vector $\sigma_{\mathrm{s}}$ of layer $K \in[1, N]$ is then defined as

$\boldsymbol{\sigma}_{\mathrm{s}}=\mathbf{P}_{K} \boldsymbol{\beta}_{K}$ 
where $\mathbf{P}_{K}$ denotes a matrix of stress interpolation functions

$\mathbf{P}_{K}=\left[\begin{array}{cccccc}F_{0} & 0 & F_{1} & 0 & F_{2} & 0 \\ 0 & F_{0} & 0 & F_{1} & 0 & F_{2}\end{array}\right]$

which are given as

$$
\begin{aligned}
& F_{0}=-\frac{1}{4}+\frac{\zeta_{K}}{2}+\frac{3}{4} \zeta_{K}^{2} \\
& F_{1}=\frac{3\left(1-\zeta_{K}^{2}\right)}{2 h_{K}} \\
& F_{2}=-\frac{1}{4}-\frac{\zeta_{K}}{2}+\frac{3}{4} \zeta_{K}^{2}
\end{aligned}
$$

and $\boldsymbol{\beta}_{K}$ is a vector of six unknown stress parameters

$\boldsymbol{\beta}_{K}=\left\{\sigma_{K}^{13, \text { top }}, \sigma_{K}^{23, \text { top }}, R_{K}^{13}, R_{K}^{23}, \sigma_{K}^{13, \text { bot }}, \sigma_{K}^{23, \text { bot }}\right\}^{\mathrm{T}}$

The subscripts 'top' and 'bot' in (35) refer to the top edge (at $\xi_{K}=h_{K} / 2$ ) and the bottom edge (at $\left.\xi_{K}=-h_{K} / 2\right)$ of layer $K$, respectively, and

$R_{K}^{\alpha 3}=\int_{-h_{K} / 2}^{h_{K} / 2} \sigma^{\alpha 3} \mu \mathrm{d} \xi_{K}$

are the transverse shear resultants of layer $K$. Note that nondimensional layer coordinate $\zeta_{K}=\xi_{K} /\left(h_{K} / 2\right) \in$ $[-1,1]$ was introduced in (34).

Now assembling the unknown stress parameters through a laminate thickness in a vector $\boldsymbol{\beta}$

$\boldsymbol{\beta}_{(6 \times N)}=\sum_{K=1}^{N} \boldsymbol{\beta}_{K}$

concluding that $\gamma=\gamma(\mathbf{u})$ and $\tilde{\gamma}=\tilde{\gamma}(\mathbf{u})$, and using the underlined part of the functional (31) we may express the stress parameters $\boldsymbol{\beta}$ as functions of the kinematic parameters $\mathbf{u}$

$\boldsymbol{\beta} \rightarrow$ Reissner's functional $(31) \rightarrow \boldsymbol{\beta}(\mathbf{u})$

In this process the continuity of the interlaminar shear stresses can naturally be satisfied by employing the following conditions

$\begin{array}{ll}\sigma_{(K+1)}^{\alpha 3, \text { bot }}=\sigma_{K}^{\alpha 3, \text { top }} & \text { if } K \in[1, N-1] \\ \sigma_{K}^{\alpha 3, \text { bot }}=\bar{\sigma}^{\alpha 3, \text { bot }} & \text { if } K=1 \\ \sigma_{K}^{\alpha 3, \text { top }}=\bar{\sigma}^{\alpha 3, \text { top }} & \text { if } K=N\end{array}$

where $\bar{\sigma}^{\alpha 3, \text { bot }}$ and $\bar{\sigma}^{\alpha 3 \text {,top }}$ are applied stresses at the bottom and at the top surface of the shell, respectively. We end up with a vector $\tilde{\boldsymbol{\beta}}(\mathbf{u})$

$\boldsymbol{\beta}(\mathbf{u}) \rightarrow$ Equations $(39) \rightarrow \tilde{\boldsymbol{\beta}}(\mathbf{u})$

which has dimension of $6+(N-1) \times 4$. The transverse shear stresses of a particular layer $K$

layer $K: \boldsymbol{\sigma}_{\mathrm{s}}(\mathbf{u})=\mathbf{P}_{K} \tilde{\boldsymbol{\beta}}_{K}(\mathbf{u})$

are then obtained by locating the corresponding stress parameters in $\tilde{\boldsymbol{\beta}}(\mathbf{u})$ $\tilde{\boldsymbol{\beta}}(\mathbf{u}) \rightarrow$ Identifying the terms related to layer

$K \rightarrow \tilde{\boldsymbol{\beta}}_{K}(\mathbf{u})$

It can be shown (see [5]) that the integration of the transverse shear stresses through a particular layer results in a sum of two parts

$\int_{-h_{K} / 2}^{h_{K} / 2} \boldsymbol{\sigma}_{\mathrm{s}} \mu \mathrm{d} \xi_{K}=\int_{-h_{K} / 2}^{h_{K} / 2} \mathbf{P}_{K} \tilde{\boldsymbol{\beta}}_{K}(\mathbf{u}) \mu \mathrm{d} \xi_{K}=\mathbf{Q}_{K}^{0} \boldsymbol{\gamma}+\mathbf{Q}_{K}^{1} \tilde{\boldsymbol{\gamma}}$

where $\mathbf{Q}_{K}^{0}$ and $\mathbf{Q}_{K}^{1}$ are variationally obtained constitutive matrices following from the Reissner's functional. Now a new form of submatrices in (29) relating the transverse shear forces $\mathbf{q}$ and $\tilde{\mathbf{q}}$ with the transverse shear strains $\gamma$ and $\tilde{\gamma}$ are defined as

$\mathbf{Z}_{0}=\sum_{K=1}^{N} \mathbf{Q}_{K}^{0}, \quad \mathbf{Z}_{1}=\sum_{K=1}^{N} \mathbf{Q}_{K}^{1}, \quad \mathbf{Z}_{2}=\sum_{K=1}^{N} g_{K} \mathbf{Q}_{K}^{1}$

Details of the procedure described in this section can be found in [5].

\subsection{Finite element interpolation and implementation}

The above laminated shell model is transformed into an isoparametric finite element in a usual way (see e.g. [3]). Convective coordinates $\xi^{1}$ and $\xi^{2}$ from the previous sections are replaced with isoparametric coordinates of a shell finite element. According to the isoparametric concept, we use standard interpolation functions to define shell geometry within one element as

$\mathbf{X}^{0}=\sum_{a=1}^{n_{\mathrm{en}}} N_{a}\left(\xi^{1}, \xi^{2}\right) \mathbf{X}_{a}^{0}, \quad \mathbf{T}=\sum_{a=1}^{n_{\mathrm{en}}} N_{a}\left(\xi^{1}, \xi^{2}\right) \mathbf{T}_{a}$

where $n_{\mathrm{en}}$ is the number of element nodes, $N_{a}: \square \rightarrow \mathbb{R}$ are the corresponding shape functions, whereas $(\circ)_{a}$ are the corresponding nodal values. Displacements are interpolated as

$\mathbf{u}^{0}=\sum_{a=1}^{n_{\mathrm{en}}} N_{a}\left(\xi^{1}, \xi^{2}\right) \mathbf{u}_{a}^{0}, \quad \mathbf{w}=\sum_{a=1}^{n_{\mathrm{en}}} N_{a}\left(\xi^{1}, \xi^{2}\right) \mathbf{w}_{a}$

and the shell director at the deformed configuration is given as

$\mathbf{t}=\sum_{a=1}^{n_{\mathrm{en}}} N_{a}\left(\xi^{1}, \xi^{2}\right) \mathbf{t}_{a}\left(\alpha_{a}^{1}, \alpha_{a}^{2}\right)$

Virtual quantities are interpolated in the same way and derivatives of the interpolated quantities with respect to the $\xi^{\alpha}$ coordinates are obtained trivially. To avoid the shear locking we use the assumed natural strain (ANS) method as suggested by Bathe and Dvorkin [1]. Accordingly, the transverse shear strains for $n_{\mathrm{en}}=4$ are interpolated in $\xi^{1}, \xi^{2}, \xi$ coordinates as 
$\gamma_{13}=\frac{1}{2}\left[\left(1-\xi^{2}\right) \gamma_{13}^{B}+\left(1+\xi^{2}\right) \gamma_{13}^{D}\right]$

$\gamma_{23}=\frac{1}{2}\left[\left(1-\xi^{1}\right) \gamma_{23}^{A}+\left(1+\xi^{1}\right) \gamma_{23}^{C}\right]$

$\tilde{\gamma}_{13}=\frac{1}{2}\left[\left(1-\xi^{2}\right) \tilde{\gamma}_{13}^{B}+\left(1+\xi^{2}\right) \tilde{\gamma}_{13}^{D}\right]$

$\tilde{\gamma}_{23}=\frac{1}{2}\left[\left(1-\xi^{1}\right) \tilde{\gamma}_{23}^{A}+\left(1+\xi^{1}\right) \tilde{\gamma}_{23}^{C}\right]$

where the strains $\gamma_{i 3}^{(\circ)}$ and $\tilde{\gamma}_{i 3}^{(\circ)}$ are evaluated at the point (o) in accordance with the expressions derived in the previous sections. Positions of the mid-side points $A, B$, $C, \quad D$ are $\mathbf{X}^{0, L}=\frac{1}{2}\left[\mathbf{X}^{0, M}+\mathbf{X}^{0, N}\right]$, where $(L, M, N) \in$ $\{(A, 1,2),(B, 2,3),(C, 3,4),(D, 1,4)\}$.

\section{Numerical examples}

In this section numerical results obtained by the above described finite element formulation are compared with exact analytical solutions.

\subsection{Cylindrical bending of a shell panel}

Consider a simply supported, three-layered, crossply $\left[90^{\circ} / 0^{\circ} / 90^{\circ}\right]$ laminated cylindrical panel of infinite length, loaded by sinusoidally distributed pressure $\bar{q}=q \sin (\pi a / b)$, where $a \in[0, b]$, over the top surface (Fig. 2). The geometry is defined by $R / b=3 / \pi$, where $R$ is radius of the panel and $b$ is its arc length in the circumferential direction. The material properties are

$E_{1}=25.0, \quad E_{2}=1.0, \quad G_{12}=G_{13}=0.5$,

$G_{23}=0.2, \quad v_{12}=0.25$

where 1 defines the fiber direction; 2 the transverse direction; 3 the thickness direction and $v_{12}$ the major Poisson's ratio. Analytical solution of this problem was given by Ren [18].

The following nondimensional quantities are shown in Figs. 3-13: normalized "in-plane" displacement $v=$ $\bar{u}_{2}=u_{2} E_{\mathrm{T}} h^{2} /\left(q a^{3}\right)$ at $a=0$, normalized transverse displacement $w=\bar{u}_{3}=u_{3} 10 E_{\mathrm{T}} h^{3} /\left(q R^{4}\right)$ at $a=b / 2$, normalized circumferential stress $S 2=\bar{\sigma}^{22}=\sigma^{22} h^{2} /\left(q R^{2}\right)$ at $a=b / 2$ and normalized transverse shear stress $S 4=$ $\bar{\sigma}^{23}=\sigma^{23} h / q R$ at $a=0$. Finite element solutions (denoted as RMZC) are obtained with a mesh of $1 \times 40$ elements for one-half of the structure. Figs. 3 and 4

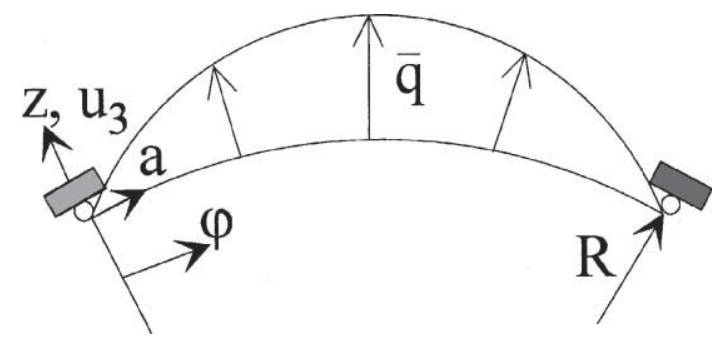

Fig. 2. Shell panel: geometry and loading.

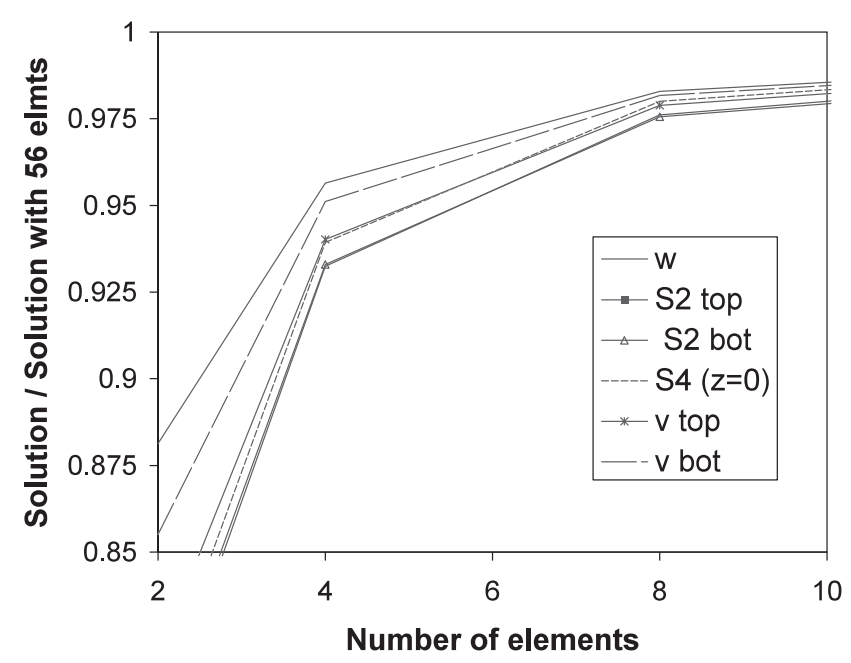

Fig. 3. Shell panel: convergence of displacements and stresses for $R / h=4$.

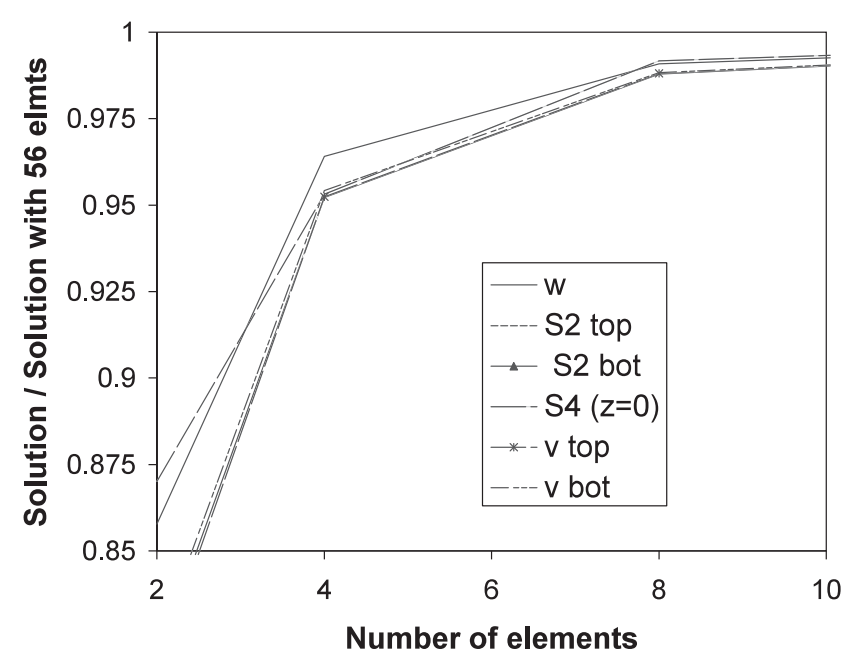

Fig. 4. Shell panel: convergence of displacements and stresses for $R / h=100$.

show a convergence of numerical results. Figs. 5-13 show through-the-thickness distribution of displacements and stresses. The RMZC prediction of $v$ and $w$ displacements is close to the analytical solutions. Figs. 7, 10 and 13 show the transverse shear stresses calculated from the constitutive equations without using shear correction factors. The agreement of RMZC results with analytical solutions is reasonable.

\subsection{Cylindrical shell}

Ten-layered $\left[90^{\circ} / 0^{\circ} / 90^{\circ} / 0^{\circ} / 90^{\circ}\right]_{S}$ cylindrical shell of thickness $h$ is defined by ratio $a / R=4$, where $a$ is the length of the cylinder and $R$ is its radius (Fig. 14). Thickness of each layer is $h / 10$. The $0^{\circ}$ layer fibers are parallel to the longitudinal coordinate $x$. The cylinder is 


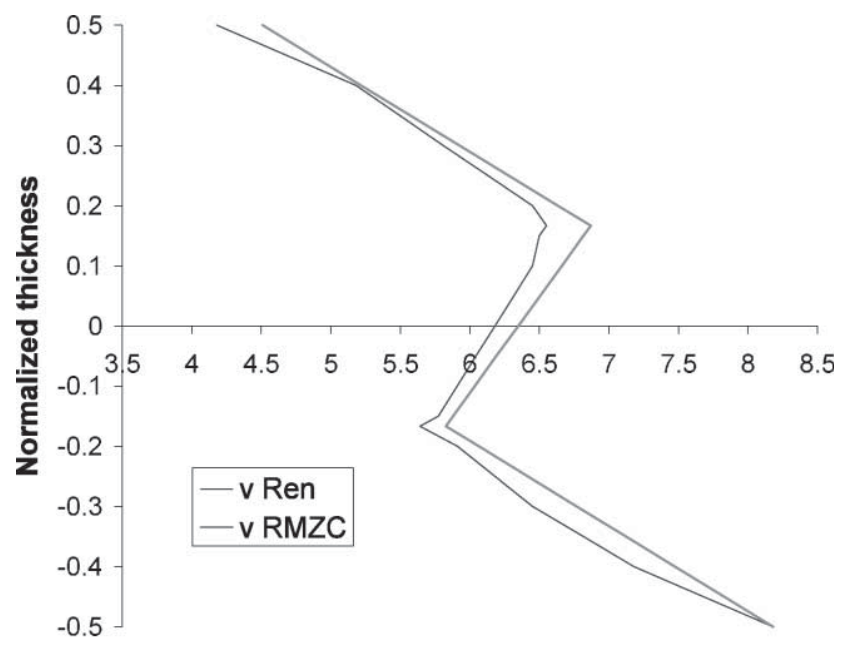

Normalized displacement

Fig. 5. Shell panel: normalized through-the-thickness displacements in the direction of the curvilinear coordinate $a$ for $R / h=4$.

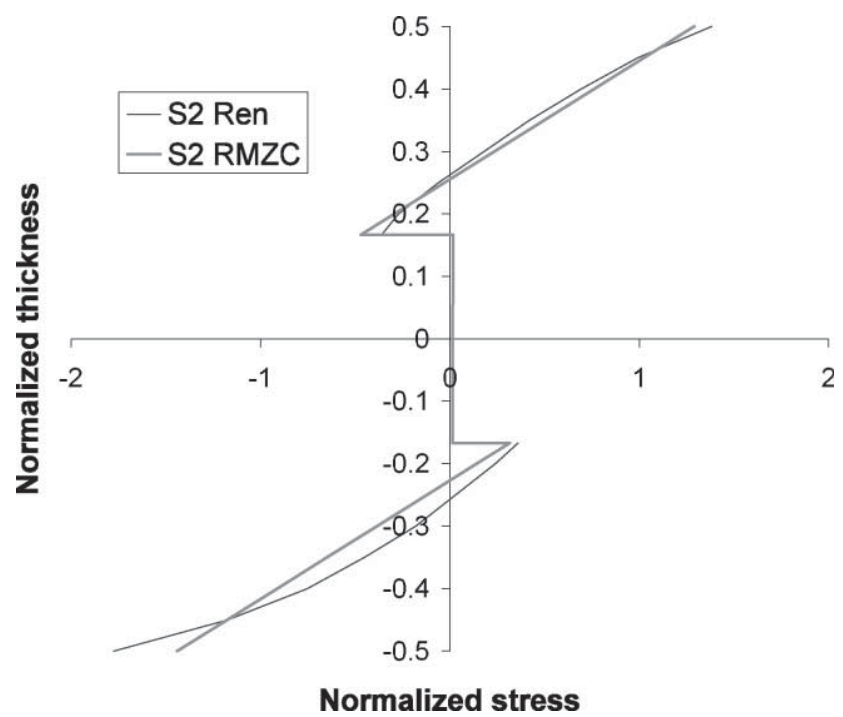

Fig. 6. Shell panel: normalized "in-plane" stresses for $R / h=4$.

supported by the shear diaphragms at both ends and subjected to the transverse pressure $q$ distributed on the shell internal surface as

$\bar{q}=q \sin \frac{\pi x}{a} \cos 4 \theta$

where $x \in[0, a]$ and $\theta \in[0,2 \pi]$. Material characteristics are

$E_{\mathrm{L}} / E_{\mathrm{T}}=25.0, \quad G_{\mathrm{LT}} / E_{\mathrm{T}}=0.5$,

$G_{\mathrm{TT}} / E_{\mathrm{T}}=0.2, \quad v_{\mathrm{LT}}=0.25$

Analytical solution of this problem was given by Varadan and Bhaskar [21].

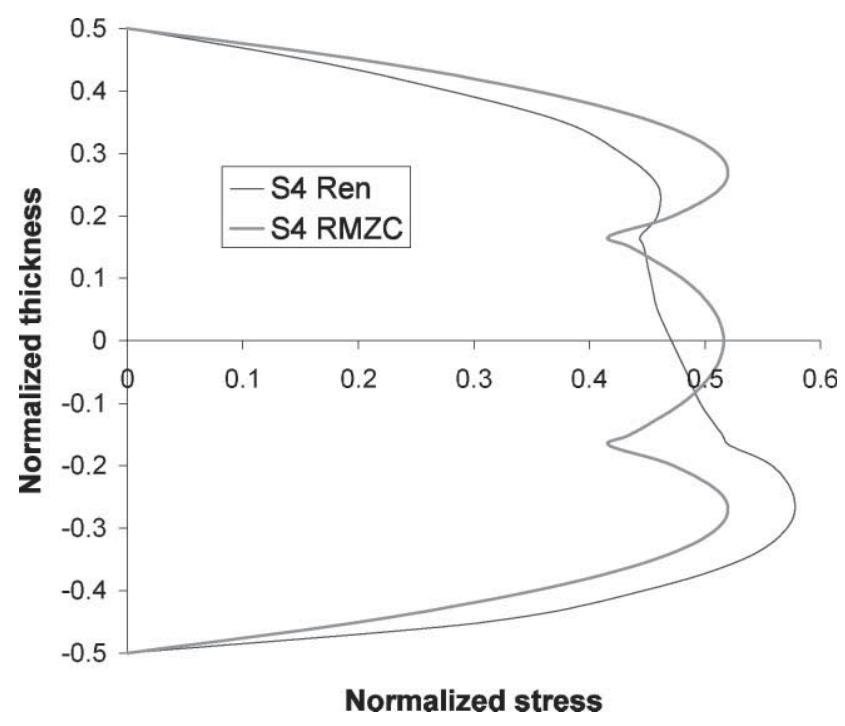

Fig. 7. Shell panel: normalized transverse shear stresses for $R / h=4$.

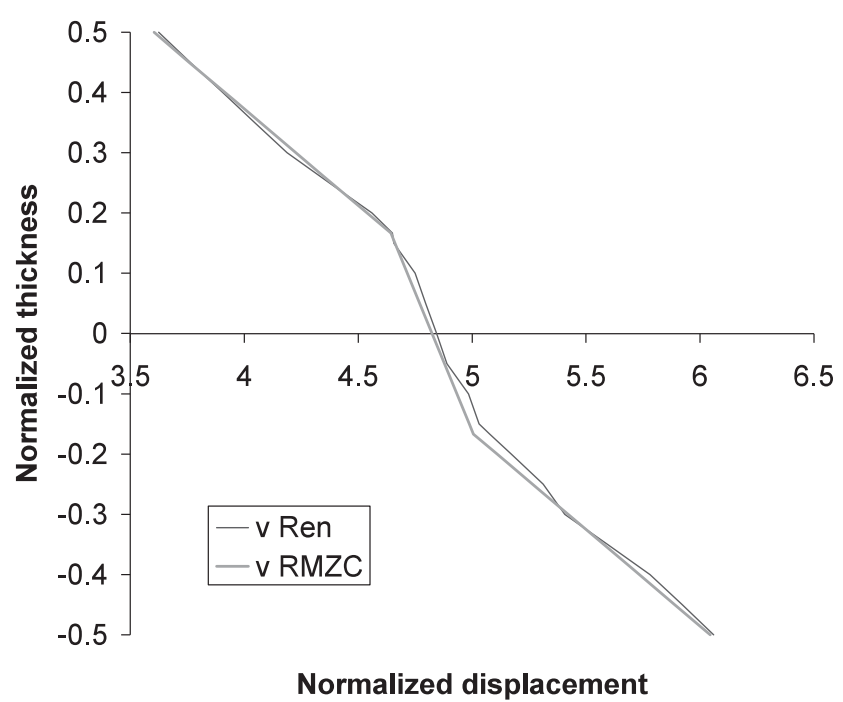

Fig. 8. Shell panel: normalized through-the-thickness displacements in the direction of the curvilinear coordinate $a$ for $R / h=10$.

Due to the symmetry only one-eight of the cylinder $(\theta \in[0, \pi / 2]$ and $x \in[0, a / 2])$ is discretized by finite elements. Discretization is done by $16 \times 40$ elements, where 40 elements are used in the circumferential direction. Boundary conditions are $u_{1} \neq 0, u_{2}=u_{3}=0$, where $u_{1}$ is longitudinal displacement, $u_{2}$ is circumferential displacement and $u_{3}$ is transverse displacement.

Figs. 15 and 16 present nondimensional quantities $v=\bar{u}_{2}=u_{2} 10 E_{\mathrm{L}} h^{2} /\left(q R^{3}\right)$ at $(x, \theta)=(a / 2, \pi / 8)$, and $S_{z b}=\bar{\sigma}_{23}=\sigma_{23} 10 h /(q R)$ at the closest integration point to $(x, \theta)=(a / 2, \pi / 8)$. The results obtained by the $\mathrm{RMZC}$ finite elements are in reasonable agreement with the analytical solution. 


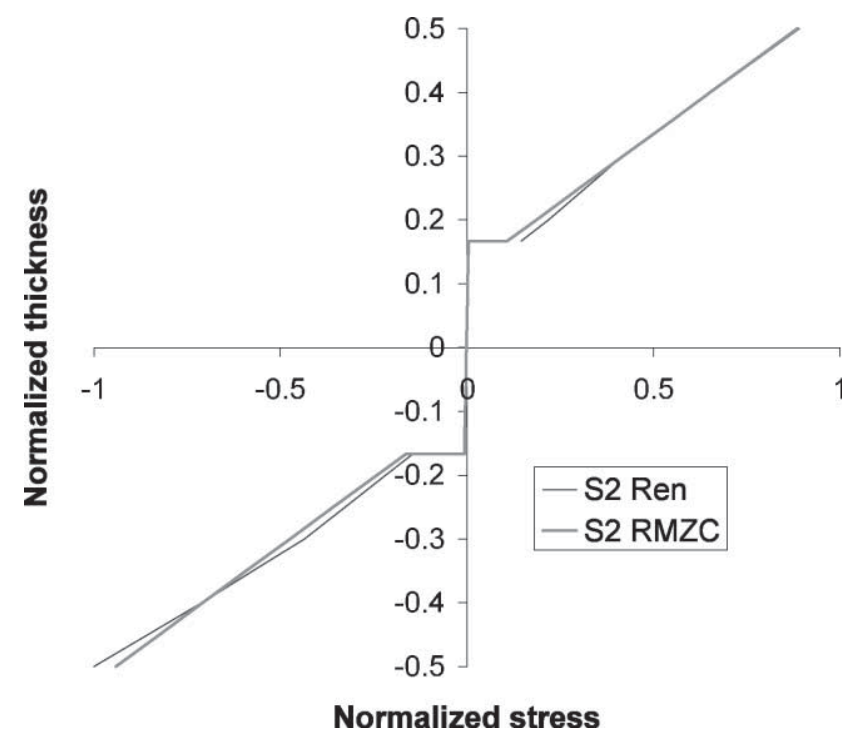

Fig. 9. Shell panel: normalized "in-plane" stresses for $R / h=10$.

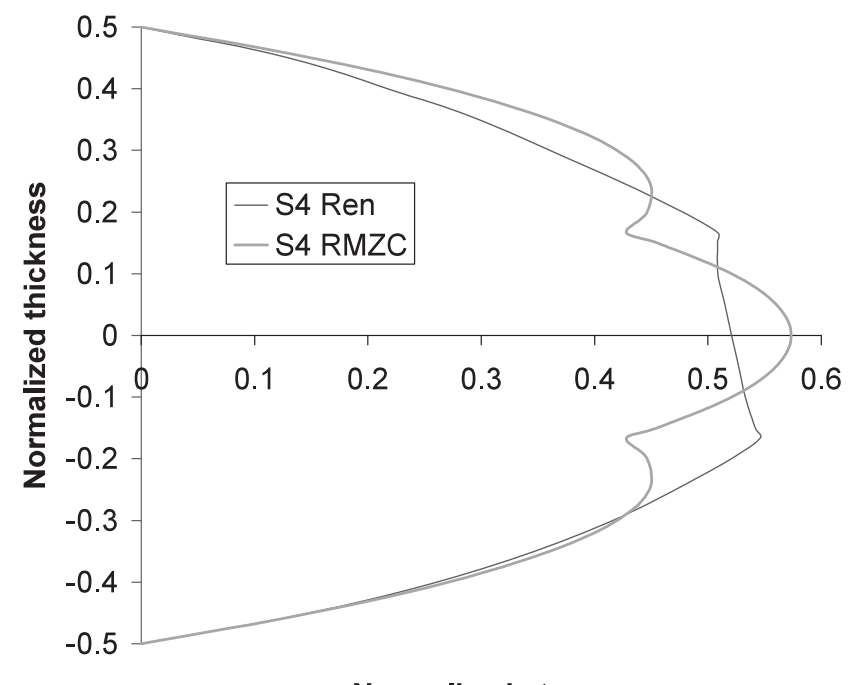

Normalized stress

Fig. 10. Shell panel: normalized transverse shear stresses for $R / h=10$.

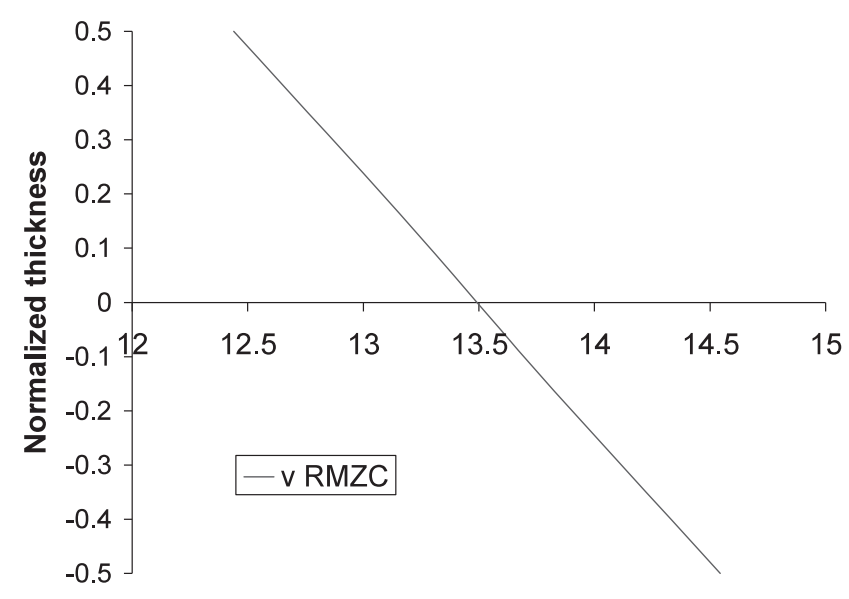

Normalized displacement

Fig. 11. Shell panel: normalized through-the-thickness displacements in the direction of the curvilinear coordinate $a$ for $R / h=50$.

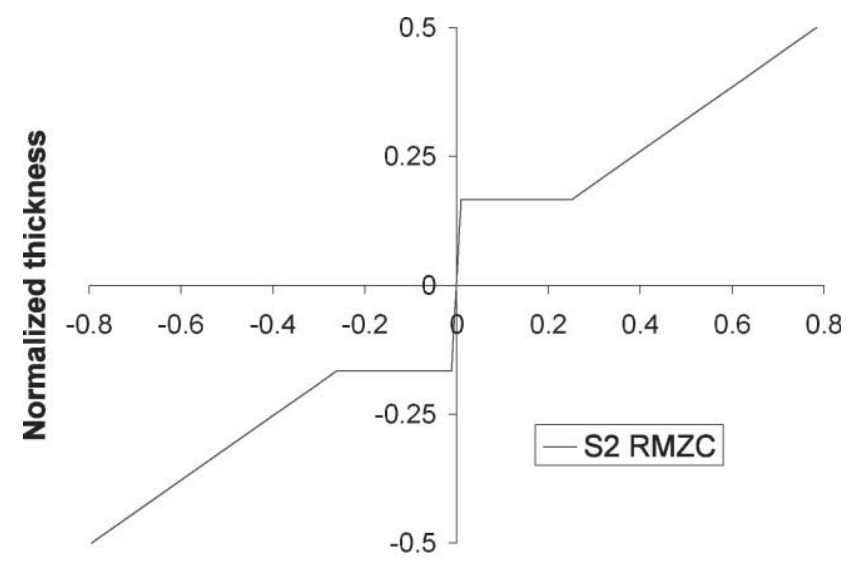

Normalized stress

Fig. 12. Shell panel: normalized "in-plane" stresses for $R / h=50$.

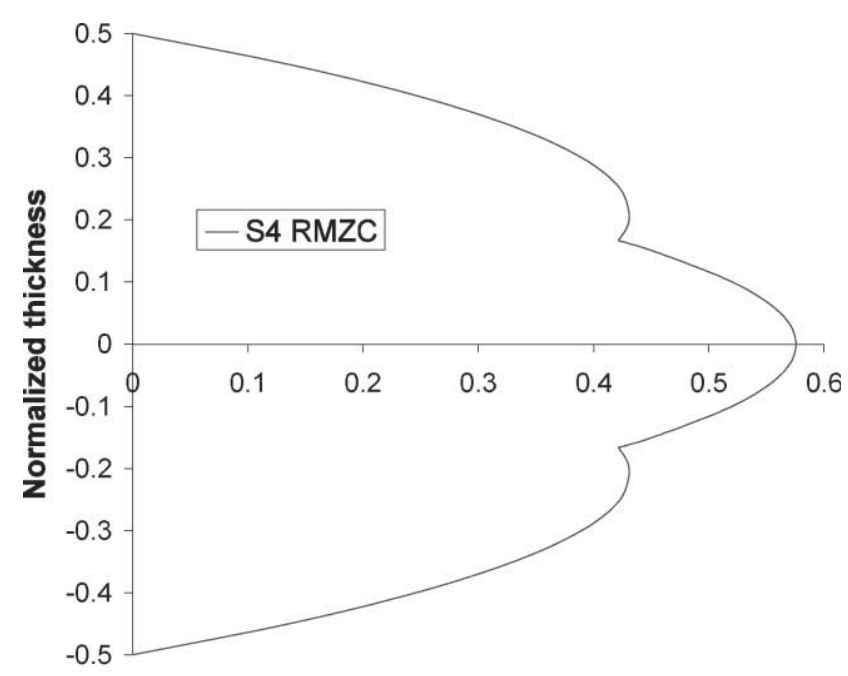

Normalized stress

Fig. 13. Shell panel: normalized transverse shear stresses for $R / h=50$.

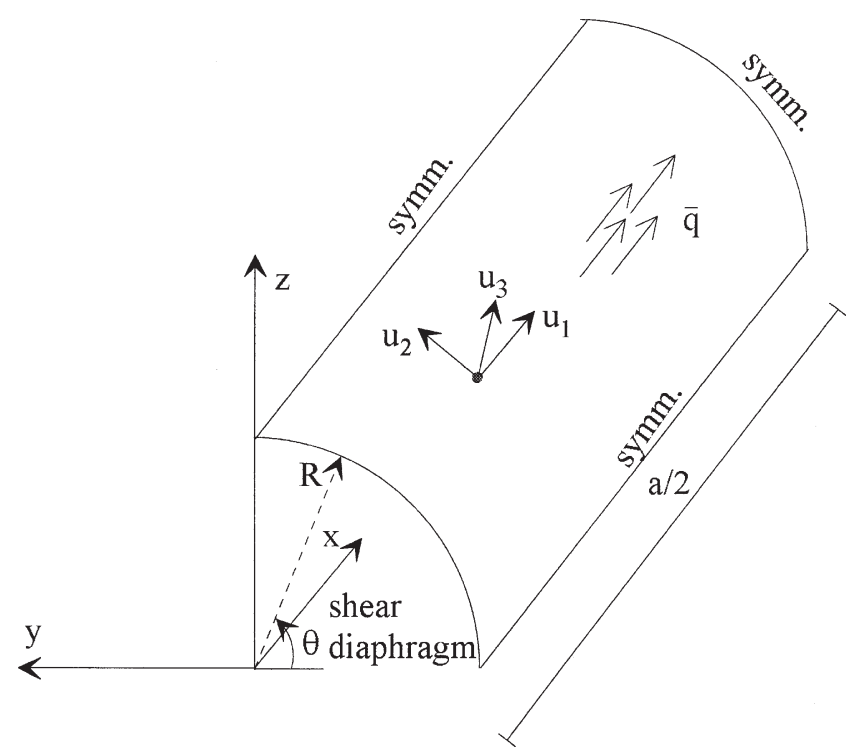

Fig. 14. Cylindrical shell: geometry and loading. 


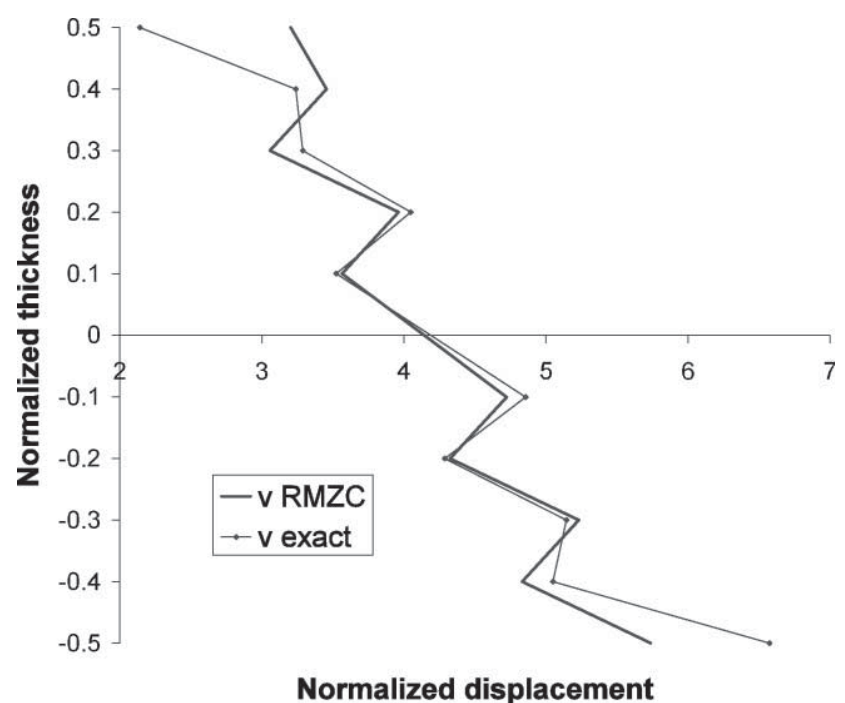

Fig. 15. Cylindrical shell: normalized through-the-thickness displacements for $R / h=4$.

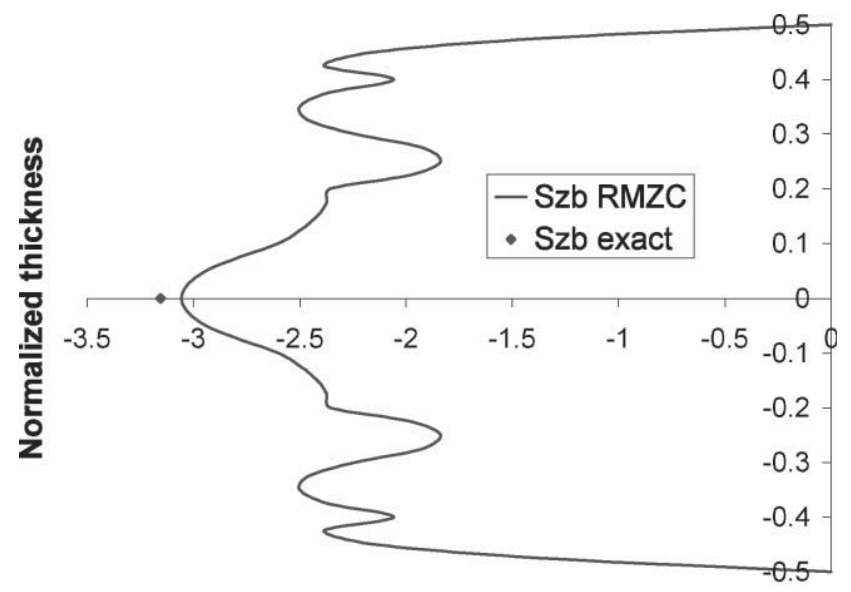

Normalized stress

Fig. 16. Cylindrical shell: normalized transverse shear stresses for $R / h=4$.

\section{Conclusions}

This work elaborated upon the composite shell formulation with a zig-zag through-the-thickness distribution of displacements and interlaminar continuity of transverse shear stresses. First the theoretical issues were clarified to provide a solid basis for the finite element implementation. In numerical examples special attention was given to comparison of the transverse shear stresses calculated from the constitutive equations with the available analytical solutions. A reasonable agreement was found although the effect of through-the-thickness deformation was not explicitly considered.

The model considered cannot predict without postprocessing neither the transverse normal stress nor an unsymmetrical distribution of the transverse shear stresses. It is believed, however, that by using recent concepts of modern shell models (see e.g. [7]) the present formulation can be extended to include an accurate through-the-thickness description of both normal and transverse stresses.

\section{References}

[1] Bathe K-J, Dvorkin E. A four node plate bending element based on Mindlin/Reissner plate theory and mixed interpolation. Int $\mathbf{J}$ Numer Meth Eng 1985;21:367-83.

[2] Bhaskar K, Varadan TK. Reissner's new mixed variational principle applied to laminated cylindrical shells. J Pressure Vessel Technol 1992;114:115-9.

[3] Brank B, Perić D, Damjanić FB. On implementation of a nonlinear four-node shell finite element for thin multilayered elastic shells. Comput Mech 1995;16:341-59.

[4] Brank B, Ibrahimbegović A. On the relation between different parametrizations of finite rotations for shells. Eng Comput 2001;18:1091-107.

[5] Brank B, Carrera E. Multilayered shell finite element with interlaminar continuous shear stresses: A refinement of the Reissner-Mindlin formulation. Int J Numer Meth Eng 2000;48:84374.

[6] Brank B, Carrera E. A family of shear-deformable shell finite elements for composite structures. Comput Struct 2000;76:287-97.

[7] Brank B, Korelc J, Ibrahimbegović A. Nonlinear shell problem formulation accounting for through-the-thickness stretching and its finite element implementation. Comput Struct 2002;80: 699-717.

[8] Carrera E. $C^{0}$ Reissner-Mindlin multilayered plate elements including zig-zag and interlaminar stresses continuity. Int J Numer Meth Eng 1996;39:1797-820.

[9] Carrera E. A study of transverse normal stress effect on vibration of multilayered plates and shells. J Sound Vibr 1999;225:803-29.

[10] Cho YB, Averil RC. First-order zig-zag sublaminate plate theory and finite element model for laminated composite and sandwich panels. Compos Struct 2000;50:1-15.

[11] Di S, Rothert H. Solution of a laminated cylindrical shell using an unconstrained third-order theory. Comput Struct 1998:291-303.

[12] Di S, Ramm E. Hybrid stress formulation for higher-order theory of laminated shell analysis. Comput Meth Appl Mech Eng 1993;109:359-76.

[13] Icardi U. Eight-noded zig-zag element for deflection and stress analysis of plates with general lay-up. Compos Part B 1998;29B:425-41.

[14] Jing H, Tzeng K. Refined shear deformation theory of laminated shells. Amer Inst Aeronaut Astronaut J 1993;31:765-73.

[15] Murakami H. Laminated composite plate theory with improved in-plane response. J Appl Mech 1986;53:661-6.

[16] Noor AK, Burton WS, Bert CW. Computational models for sandwich panels and shells. Appl Mech Rev 1996;9:155-99.

[17] Reddy JN. Mechanics of laminated composite plates: theory and analysis. CRC Press; 1997.

[18] Ren JG. Exact solutions for laminated cylindrical shells in cylindrical bending. Compos Sci Eng 1987;29:169-87.

[19] Di Schuva M. Bending, vibration and buckling of simplysupported thick multilayered orthotropic plates. An evaluation of a new displacement model. J Sound Vibr 1986;105:425-42.

[20] Toledano M, Murakami H. A high-order laminated plate theory with improved in-plane responses. Int J Solids Struct 1987;23: 111-31.

[21] Varadan TK, Bhaskar K. Bending of laminated orthotropic cylindrical shells-an elasticity approach. Compos Struct 1991;17:141-56. 